\title{
Der Betrieb als Projekthaus - Wie Werkverträge die Arbeitswelt verändern
}

\author{
SANDRA SIEBENHÜTER
}

\begin{abstract}
„Make or buy?“ Diese Frage wird in vielen privatwirtschaftlichen und öffentlichen Organisationen vermehrt gestellt. Nach betriebswirtschaftlicher Lesart sind Werkverträge (WV) unter finanziellen, strategisch operativen und sogar sozialen Aspekten für Unternehmen attraktiver als die ausschließliche Erledigung der anfallenden Arbeitsumfänge mit Stammbeschäftigten bzw. Leiharbeitnehmern. ${ }^{1}$ Durch die Vergabe von Werkverträgen kann sich der Betrieb in mehrfacher Hinsicht sozialer, sozialpartnerschaftlicher sowie tarifvertraglicher Verantwortung entledigen und Konflikte mit Betriebsräten und Stammbeschäftigten minimieren.
\end{abstract}

Die im Folgenden präsentierten Erkenntnisse beruhen auf einer empirischen Studie, ${ }^{2}$ in deren Rahmen die quantitative Verbreitung von Werkverträgen u. a. unter Einbeziehung des IAB-Betriebspanels, Umfragen von Einzelgewerkschaften und Sonderauswertungen des Bayerischen Landesamtes für Statistik sowie des Gewerbeamtes der Stadt München analysiert wurde. Zusätzlich wurden durch mehr als 40 leitfadengestützte Interviews weitergehende qualitative Informationen u. a. über Einsatzstrategien von Werkverträgen, Beschäftigungsbedingungen bei Werkvertragsfirmen wie auch über Auswirkungen beim Werkbesteller und über eine höchst dynamische Beratungsindustrie gewonnen. Schließlich wurde eine explorative Kurzstudie ${ }^{3}$ über das Verhältnis von Entwicklungsdienstleistern und Endherstellern (OEMs: Original Equipment Manufacturers) hinzugezogen.
Besonders verbreitet sind Werkverträge in der Bauindustrie, wo Großprojekte im Umfang mehrerer Hundert Millionen Euro von einem Generalunternehmer durchgeführt werden, der oft nur wenige oder keine eigenen Baufacharbeiter beschäftigt. Dem Generalunternehmer kommt die Aufgabe zu, Hunderte, zeitlich dem Bauprozess angepasste Gewerke über Subunternehmen durchzuführen und das Projekt in seiner Gesamtheit zu steuern. Verbreitung findet dieses Modell inzwischen auch in der Automobilindustrie: Im Leipziger BMW-Werk ${ }^{4}$ sind neben ca. 3.500 Stammbeschäftigten noch ca. 1.800 Leiharbeiter und 2.200 Mitarbeiter von ca. 20 Fremdfirmen in den Werkshallen tätig, wofür es u. a. als „Beste Europäische Fabrik 2013“ ausgezeichnet wurde. In der Begründung hierzu heißt es: „Pluspunkte sammelte das Werk auch durch seine integrierte und flexible Wertschöpfungskette, innovative Produkt- und Prozessentwicklungen sowie eine breite Mitarbeitermobilisierung mit einer vorbildlichen Leistungskultur". ${ }^{\text {. }}$

Das Modell des "projektorientierten Unternehmens“ ist nach Meinung vieler Unternehmensberater und Manager zukunftsweisend, da es das Risiko volatiler Märkte begrenzt. Projekte sind in diesem Arrangement nicht mehr lediglich ein Anhängsel des Unternehmens, sondern bil-

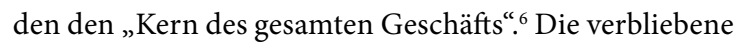
Rumpfmannschaft verfügt vor allem über organisationale Metakompetenzen und übernimmt in erster Linie Steuerungs-, Organisations- und Kontrollaufgaben. Die Fest-
1 Vgl. Nienhüser, W./Baumhus, W. (2002): Fremd im Betrieb: Der Einsatz von Fremdfirmenpersonal als Arbeitskräftestrategie, in: Martin, A./Nienhüser, W. (Hrsg.): Neue Formen der Beschäftigung - neue Personalpolitik? München, S. 78-90; Mühlbacher, A./Pflügel, R. (2008): Strategien des Outsourcing: Das (digitale) Krankenhaus zwischen Integration und Fokussierung, Berlin, S. 15.

2 Siebenhüter, S. (2013): Report: Werkverträge in Bayern Das neue Lohndumpinginstrument, DGB Bayern, München, http://bayern.dgb.de/themen/++co++baba127a-f90211 e2-b5e9-00188b4dc422.

3 Vgl. Siebenhüter, S./MeyerT. (2012): Innovationsdruck vs. Risikostreuung: Das Dilemma eingekauften Wissens von Entwicklungsdienstleistungen für Automobilhersteller, Arbeitspapier, Otto-Brenner-Stiftung, Frankfurt a. M.: http://www.otto-brenner-shop.de/fileadmin/user_data/ shop/dokumente/Arbeitspapiere/S7H_Innovationsruck.pdf.

4 Vgl. Wirtschaftswoche (2013): Autobauer - Werkverträge sind schlummernde Zeitbomben, vom 12.09.: http://www.wiwo.de/unternehmen/industrie/autobauerwerkvertraege-sind-schlummernde-zeitbomben/ 8750794.html.

5 Vgl. 10. WirtschaftsWoche Konferenz 2013: http://www.euroforum.de/news/BMW-Werk-Leipzig-ist-die \%E2\%80\%9E Beste-Europaeische-Fabrik\%E2\%80\% 9C_93859.

6 Vgl. Bea, F.-X./Scheurer, S. (2011): Trends im Projektmanagement. Vom Management von Projekten zum projektorientierten Unternehmen, in: Zeitschrift Führung + Organisation $80(6)$, S. $425-431$. 
anstellung von Mitarbeiterinnen und Mitarbeitern wird daher zukünftig die Ausnahme sein. ${ }^{7}$

\section{Sieben Belegschaften, sieben Statusgruppen - ein Werkstor}

Schon heute ergibt sich bei völliger Ausschöpfung der unternehmerischen Gestaltungsfreiheit (ohne Einbeziehung weiterer Subunternehmen) seitens des Werkbestellers und des Werknehmers folgendes Bild (Abbildung. 1):

Ein direkter Einstieg in ein "Premiumunternehmen“ (1A-Arbeitsplatz) gelingt vielfach nur noch über einen Ausbildungsplatz, in Einzelfällen über sehr spezifische und damit seltene Fachqualifikationen im Hochqualifiziertenbereich und eingeschränkt über den Weg einer Befristung (1. Stufe). Zusätzliche Chancen bieten, je nach Qualifikationsebene, auch Brückenein- und aufstiege aus den Stufen 2-6. Dass, wie in den Interviews mehrfach berichtet, ${ }^{8}$ ein Stammarbeitsplatz bei einem Dienstleister (3. Stufe) aufgegeben wird, um als Leiharbeitnehmer (2. Stufe) (vielfach mit Branchenzuschlägen) beim Werkbesteller zu arbeiten, zeigt die Verwerfungen dieses Systems. Das Konstrukt eines „sicheren Kerns“ und weniger sicheren Satellitenbelegschaften ist vielfach durch eine Hierarchie von sozialen und tariflichen Rechten charakterisiert und erzeugt sowohl innerhalb wie auch zwischen den verschiedenen Belegschaften soziale Spannungen in Form von Konkurrenz, Dominanzstreben und Gefühlen der Über- und Unterlegenheit.

Bisher verbreitete interne Bewährungsaufstiege beim Werkbesteller werden abgelöst von Aufstiegen über die verschiedenen externen Statusgruppen (Stufe 1-6). ${ }^{9}$ Stets sind die Abschottungstendenzen des Werkbestellers bei gleichzeitiger Ausweitung seiner Macht- und Kontrollansprüche offen sichtbar. Die Stammbelegschaft des Werkbestellers zahlt einen hohen Preis für die Sicherheit: Sie muss die Projekte ausschreiben und vergeben, deren Durchführung zeitlich koordinieren und kontrollieren und im Falle einer Verzögerung oder eines Fehlers in kürzester Zeit nacharbeiten, um die Lücken zwischen den Einzelgewerken zu schließen und die Anschlussfähigkeit wiederherzustellen.

Die Dynamik der Fremdvergabe führt dazu, dass Ingenieure im Forschungs- und Entwicklungsbereich des Werkbestellers der Konkurrenz der Mitarbeiter vielfach tarifloser Dienstleister unterliegen und dadurch gezwungen werden, ihre Arbeitsintensität zu erhöhen. Das Risiko, durch Fremdvergabe bestimmter Tätigkeiten ihren bisherigen Arbeitsplatz (1A) zu verlieren und als Mitarbeiter eines Entwicklungsdienstleisters als Externer an ihren alten Arbeitsplatz zurückzukommen, schwebt stets über ihnen und gehört zu den nicht kalkulierbaren Risiken der zugrunde liegenden Marktlogik. Eine Studie des IMU-Instituts München unterstreicht diesen Befund, indem sie feststellt: „Die Externalisierung von Arbeitsaufgaben erfolgt zum Teil über die Externalisierung Interner. ${ }^{\text {“10 }}$ Unter Ausschöpfung aller Fle-
ABB. 1

Eine sichere Kernbelegschaft - unsichere Satellitenbelegschaften

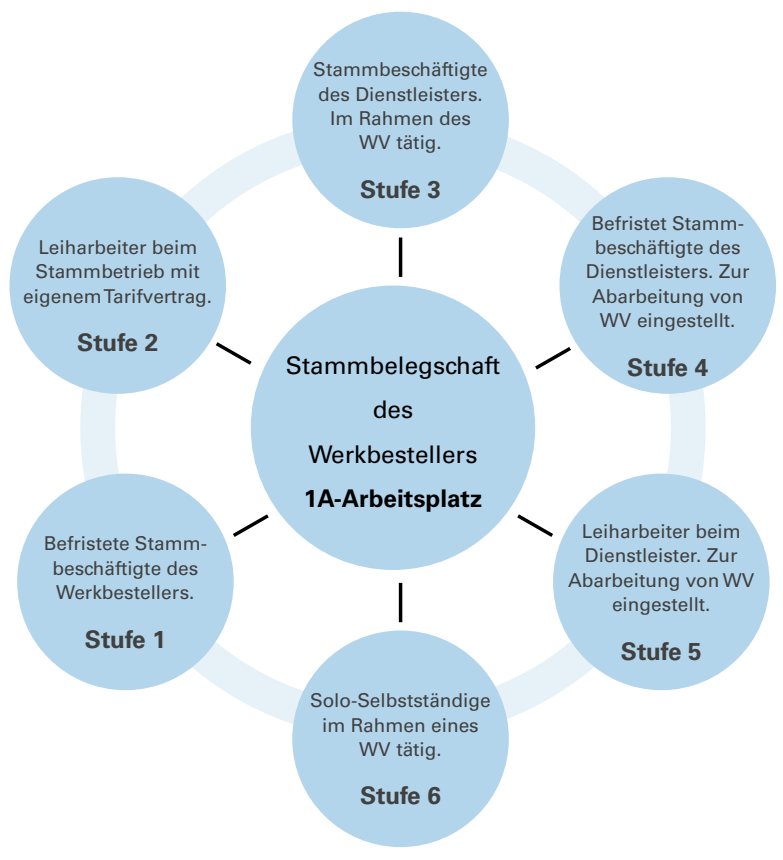

Quelle: Darstellung der Autorin (vgl. Siebenhüter 2013, S. 49).

xibilisierungsmöglichkeiten und ohne die Einbeziehung von Subunternehmen der zweiten Reihe arbeiten letztlich bis zu sieben unterschiedliche Statusgruppen parallel am Wertschöpfungsprozess des gleichen Unternehmens.

Besonders kritisch beurteilt werden muss die Vorgabe einer festgeschriebenen Flexibilitätsreserve bis zu 30 \%, da sie die langfristige Strategie der börsennotierten Unternehmen widerspiegelt. Diese fixiert die Existenz von Subbelegschaften (Stufen 1-6) und damit die dauerhafte Institutionalisierung von prekärer und atypischer

7 Vgl. Weiss, H. (2012): Amerikaner sehen in der Festanstellung ein Auslaufmodell, VDI-Nachrichten 11/2012 vom 16.03.: http://www.vdi-nachrichten.com/Technik-Gesellschaft/Amerikaner-sehen-in-Festanstellung-Auslaufmodell.

8 Vgl. Siebenhüter (2013), a.a.O., S. 26.

9 Zum strategischen Einsatz von Leiharbeit als Rekrutierungsinstrument vgl. Jahn, E./Rosholm, M. (2011): Arbeitnehmerüberlassung: In Dänemark ist Zeitarbeit ein Sprungbrett in Beschäftigung, IAB-Kurzbericht 01/2011, Nürnberg.

10 Biehler, H. (2012): Ingenieursarbeit in Bayern. Prekäre Beschäftigung auf dem Vormarsch, in: IMU Informationsdienst 1/2012, S. 59. 
ÜBERSICHT 1

\section{Arbeitsbedingungen von Berufsanfängern mit Fachhochschulstudium bei der AUDI AG und für diese tätigen Entwicklungsdienstleistern (EDL)}

\begin{tabular}{ll|l}
\hline $\begin{array}{l}\text { Arbeitsbedingungen } \\
\text { Berufsanfänger } \\
\text { FH-Ingenieur }\end{array}$ & AUDI AG & $\begin{array}{l}\text { EDL mit ca. 700 Mitarbeitern } \\
\text { im Raum Ingolstadt }\end{array}$ \\
\hline Tarif & ERA Baden Württemberg & kein Tarifvertrag \\
\hline Monatsgehalt & $\begin{array}{l}\text { ca. } 3.850-4.070 € \\
\text { (abhängig BA/Master/Dipl.) }\end{array}$ & $\begin{array}{l}3.300 € \\
\text { (nach 6 Monaten: } 3.600 €)\end{array}$ \\
\hline Urlaubstage & 30 & 30 \\
\hline Zahl der Gehälter & 13,2 & $12+1$ (freiwillige Leistung) \\
\hline Gewinnbeteiligung & $\begin{array}{l}\text { durchschnittlich 8.200 } \\
(2011)\end{array}$ & keine \\
\hline $\begin{array}{l}\text { Betriebliche } \\
\text { Altersvorsorge }\end{array}$ & $\begin{array}{l}\text { ausschließlich seitens des } \\
\text { Unternehmens finanziert }\end{array}$ & $\begin{array}{l}\text { Direktversicherung: } 48 € \\
\text { monatlich }\end{array}$ \\
\hline Arbeitszeit & 35 Stunden & 40 Stunden \\
\hline
\end{tabular}

Quelle: Siebenhüter, S. (2013): a.a.O., S. 32.
Die Folgen dieser Niedriglohnstrategie sind bekannt, ${ }^{12}$ ebenso das gemeinsame Auftreten eines hohen Beschäftigungsstandes und einer Ausbreitung sowie Verfestigung prekärer Beschäftigung. ${ }^{13}$ Der stetige Rückgang des Normalarbeitsverhältnisses (unbefristet, sozialversicherungspflichtig, in Vollzeit) von ca. $70 \%$ (2002) auf ca. $67 \%$ (2012) bei gleichzeitigem Wachstum der Beschäftigungsquote wird nachvollziehbarer, wenn man berücksichtigt, dass die Gesamtzahl der gearbeiteten Stunden in den vergangenen Jahren kontinuierlich abgenommen hat. ${ }^{14}$

\section{Das Risiko wird weitergereicht}

Die Risikoübertragung auf die Werkvertragsfirmen bleibt jedoch nicht ohne Folgen, da auch diese Abwehrstrategien entwickeln, die weit über die Beschäftigung von Leiharbeitern und befristet Beschäftigten hinausgehen.

Werkverträge für gering qualifizierte Tätigkeiten (Lager-, Verpackungs- und Hebearbeiten) mit nur geringer Wertschöpfung werden durch die Hinzuziehung von Leiharbeit oder Werkverträgen zu Niedriglöhnen über in- und ausländische Subunternehmen ausgeführt. Die Steuerung, so zeigt die hier zugrunde liegende Studie, wird durch eigens dafür gegründete $\mathrm{GmbHs}$ übernommen, die über kaum oder keine eigenen Beschäftigten verfügen. Die mit Werkverträgen einhergehende Niedriglohnpolitik ermöglicht die Umkehrung einer Befürchtung aus den 1980er Jahren (Roboter ersetzen Maschinen): Die geringen Löhne machen Neuinvestitionen in eine weitergehende Automatisierung von Verpackungsstraßen oder Hebehilfen unrentabel. ${ }^{15}$

Da bei Werkverträgen im Hochqualifiziertenbereich die Leistung und das Wissen individualisiert sind, werden konkrete Mitarbeiter oder Teams ,gekauft“. Über der Werkvertragsfirma (Ingenieurs- und Entwicklungsdienstleister) schwebt dabei die stete Gefahr der Abwerbung von Mitarbeitern bzw. Teams durch den Werkbesteller, da eingearbeitete Mitarbeiter des Dienstleisters ohne Einarbeitungszeit in die Stammbelegschaft (Stufe 1) des OEMs wechseln können.

Doch auch die Abwerbung qualifizierter Mitarbeiter/ Teams durch einen anderen Dienstleister - Abwerbeprämien von einigen Tausend Euro sind hierbei keine Seltenheit - ist

11 Siebenhüter, S./Meyer, T. (2012): a.a.0., S. 2.

12 Vgl. Destatis (2013): Datenreport 2013. Ein Sozialbericht für die Bundesrepublik Deutschland: Statistisches Bundesamt und Wissenschaftszentrum Berlin für Sozialforschung (WZB), Bonn; Kalina, T./Weinkopf, C. (2013): Niedriglohnbeschäftigung 2011, IAQ Report 01/2013, Duisburg-Essen, http://www.iaq.uni-due.de/iaq-report/2013/report2013-01.pdf.

13 Vgl. Destatis (2013): a.a.O., S. $171 \mathrm{ff}$.

14 Ebd., S. $115 f$

15 Vgl. Siebenhüter, S. (2013): a.a.O.; S. 20 und 31. 
attraktiv, da sie begehrtes Wissen über andere OEMs besitzen oder auch Türen zu möglichen neuen Kunden öffnen können. Hier ein Beispiel: Mitarbeiter/Team A ist über den Dienstleister A bei OEM X tätig; Dienstleister B ist daran interessiert, einen Auftrag von OEM X zu erhalten. Durch das Abwerben von Mitarbeiter/Team A gelingt es Dienstleister B, den Auftrag bei OEM X zu übernehmen, da der Werkvertrag des OEM vielfach nicht an eine Firma gebunden ist, sondern konkret an spezifische Personen bzw. Teams. ${ }^{16}$ Die Folge dieser individualisierten Vertragsstrategie ist eine zurückhaltende und fein austarierte Investitionsstrategie (Bezahlung, Personalentwicklung) des Dienstleisters in die eigenen Mitarbeiter. ${ }^{17}$ Aufgrund der starken Konkurrenz um Aufträge und Personal haben Mitarbeiter und eingesetzte Leiharbeiter parallel zur Abarbeitung des Werkvertrages die Aufgabe, neue Werkvertrags- und Mitarbeiterakquise zu betreiben, um ihre eigene Weiterbeschäftigung sicherzustellen. Dem Preisdruck wird vielfach durch Subwerkverträge an Soloselbstständige und ausländische Dienstleister begegnet.

Zusammenfassend zeigt sich, dass trotz diverser Unterschiede alle beteiligten Auftraggeber stets bestrebt sind, ihr unternehmerisches Risiko zu minimieren. ${ }^{18}$ Die Arbeitnehmer hingegen werden zunehmend ökonomischen Zwängen unterworfen.

\section{Die langfristigen Kosten sind noch nicht addiert}

Der kurz- und mittelfristige Erfolg von börsenorientierten Unternehmen bemisst sich an betriebswirtschaftlichen Kenngrößen und der quantitativen Erfassung von Kosten. In diesem Rationalitätskontext sind Werkverträge attraktiv, da sie als flexible Sachkosten bilanzierbar sind und ihr Einsatz zu einer Umsatzsteigerung ohne Erhöhung der Beschäftigtenzahl beiträgt. Dieses „Wachsen ohne zu wachsen“ hat unmittelbaren Einfluss auf die betriebswirtschaftlichen Kennzahlen und gilt als positives Signal an Investoren, obwohl die Erfassung zunächst finanziell nicht bezifferbarer, aber langfristiger Begleitkosten ausbleibt. Doch auch diese zunächst nur „qualitativen Kosten“ des „Projekthaus-Modells" lassen sich anhand von fünf Punkten kurz bilanzieren:

(1) Die Freiheit von Arbeitnehmern nimmt ab, da dem Prozess der funktionalen und numerischen Flexibilisierung ein ausgesprochen enger und einseitiger Freiheitsbegriff zugrunde liegt. Dieser bezieht sich auf die Freiheit der Auftraggeber, die Gewerke ausschreiben, und im Einzelfall noch auf die Freiheit von sehr hoch qualifizierten Soloselbstständigen, die ohnehin den globalen Arbeitsmarkt im Blick haben und sich über Dienstleister wie Hays, Reutax oder MBA Michael Bailey Associates auf Contracting-Basis an OEMs vermitteln lassen. Für die allermeisten Arbeitnehmer hingegen ist der Schutz eines Kollektivs und eines Tarifvertrags notwendig, da ihre Kompetenzen nicht ausreichen, um auf diesem hochgradig kompetitiven Arbeitsmarkt zu bestehen.
ÜBERSICHT 2

\section{Arbeitsbedingungen von Stationsleitungen bei der Sozialstiftung Bamberg und der hauseigenen Service $\mathbf{G m b H}$}

\begin{tabular}{l|l|l}
\hline $\begin{array}{l}\text { Arbeitsbedingungen Stations- } \\
\text { leitung mit neunjähriger } \\
\text { Berufserfahrung }\end{array}$ & $\begin{array}{l}\text { Fest angestellt über die SSB } \\
\text { in den Seniorenheimen }\end{array}$ & $\begin{array}{l}\text { Fest angestellt in der } \\
\text { Service-GmbH in den } \\
\text { Seniorenheimen }\end{array}$ \\
\hline Tarif & TVÖD & IGZ \\
\hline Monatsgehalt & ca. $2.895 €$ & $\begin{array}{l}\text { ca. } 1.900 €+300 €+200 € \\
\text { freiwillige Zulage }\end{array}$ \\
\hline Urlaubstage & Altersabhängig 29/30 Tage & $\begin{array}{l}\text { Je nach Anstellungs- } \\
\text { dauer 24-30 Tage } \\
\text { (ab dem 5. Jahr) }\end{array}$ \\
\hline Zahl der Gehälter & 12,8 & $\begin{array}{l}12+300-600 € \text { je nach } \\
\text { Dauer der Betriebszuge- } \\
\text { hörigkeit }\end{array}$ \\
\hline Wöchentliche Arbeitszeit & 39 Stunden & 35 Stunden \\
\hline Betriebliche Altersvorsorge & Ja & Nein \\
\hline
\end{tabular}

Quelle: Siebenhüter, S. (2013): a.a.O., S. 39

(2) Der Fachkräftemangel steigt an, die institutionelle Kompetenz nimmt ab. Durch die Verringerung der Wertschöpfungstiefe und die Vergabe von Einzelgewerken an zeitlich und qualifikatorisch passgenaue Dienstleister im In- und Ausland sinkt die Notwendigkeit der Ausbildungsund Weiterbildungsinvestition in die eigenen Mitarbeiter. Parallel dazu sinkt die Zahl institutioneller Schlüsselmitarbeiter, die fachliches, organisatorisches und soziales Wissen vereinen.

(3) Belegschaften, Betriebsräte und Gewerkschaften werden geschwächt, indem die Schutzfunktion des Betriebsverfassungsgesetzes umgangen wird und es zu einem Abschmelzen bzw. Einfrieren von Belegschaften in Großbetrieben kommt. Tarifauseinandersetzungen werden schwieriger, da deren Erfolg immer noch von der geschlossenen Unterstützung der Belegschaften von Großbetrieben abhängt.

(4) Die dauerhafte Ausschreibung von Gewerken birgt die Gefahr einer Billigstrategie zum Nachteil der Arbeitnehmer, der Steuerzahler und der Sozialkassen. Bei Ausschreibungen der öffentlichen Hand sollte jener mit dem wirtschaftlichsten, nicht mit dem billigsten Angebot den $\mathrm{Zu}$ -

16 Vgl. ebd., S. 33.

17 Vgl. Siebenhüter, S./Meyer, T. (2012): a.a.O., S. 9ff.

18 Über die Gründe der Inanspruchnahme von Werkverträgen vgl. Siebenhüter (2013), S. $42 \mathrm{ff}$. 
schlag erhalten. Laut Bundesrechnungshof ${ }^{19}$ lagen in $85 \%$ der Fälle jedoch gar keine Wirtschaftlichkeitsuntersuchungen vor; vielfach erhält der billigste Anbieter den $\mathrm{Zu}$ schlag. ${ }^{20}$ In der Privatwirtschaft zeigen sich ähnliche Tendenzen. Strategische Einkaufsabteilungen schreiben im Rahmen ihrer Global Sourcing-Strategie Gewerke über

19 Der Bundesrechnungshof (2013) drängt auf Wirtschaftlichkeitsuntersuchungen, Pressemitteilung vom 12. Juni, http:// www.bundesrechnungshof.de/de/veroeffentlichungen/ gutachten-berichte-bwv/pressemitteilungen/2013-presse mitteilung-05-bwv-band-18-bundesrechnungshof-draengtauf-wirtschaftlichkeitsuntersuchungen.

20 Bundesrechnungshof (2003): Gutachten des Bundesbeauftragten für Wirtschaftlichkeit in der Verwaltung für den Haushaltsausschuss des Deutschen Bundestages zur Wirtschaftlichkeit der Vergabe an Billiganbieter im Bereich des Bundesfernstraßenbaues und der Bundeshochbauten, S. 3, http://www.bundesrechnungshof.de/de/veroeffentlichungen/ gutachten-berichte-bwv/berichte/sammlung/2003-bwvgutachten-wirtschaftlichkeit-der-vergabe-an-billigstbieterim-bereich-des-bundesfernstrassenbaus-und-der-bundes hochbauten.

21 Vgl. www.mturk.com und Interview mit Six Silverman http://www.igmetall.de/SID-698F0203-57649154/internet/ docs_Turkopticon_rough_versionof_35a8a9bf34ad8f5e6e9 fee24c55bd3e26389698f.pdf. geschlossene B2B-Plattformen international aus und zwingen langjährige Kunden zu empfindlichen Preisnachlässen, um Nachfolgeaufträge zu erhalten. Besonders betroffen sind Freelancer, die sich um im Internet ausgeschriebene Aufträge im Bereich Programmierung ${ }^{21}$ oder Journalismus und Design (vgl. twago.de, clickworker.de) bewerben und im Extremfall nur bezahlt werden, wenn sie die ausgeschriebene Leistung am schnellsten und am billigsten erstellen können.

(5) Das Vertrauen in das Arbeitsrecht wird geschwächt. Die Trennung von Personalführung und Produktivarbeit verhüllt einerseits die Verantwortlichkeit von Arbeitgebern und führt zu Verunsicherung auf Mitarbeiterseite. Andererseits haben viele Werkvertragsfirmen keine Betriebsräte, die sich für die Rechte der Belegschaft einsetzen können.

\section{AUTORIN}

SANDRA SIEBENHÜTER, Dr. phil., arbeitet als Sozialwissenschaftlerin im Netzwerk Nautilus Politikberatung und als Gewerkschaftssekretärin bei der IG Metall Nürnberg. Arbeitsschwerpunkte: Arbeits- und Industriesoziologie.

sandra.siebenhueter@nautilus-politikberatung.de 